\title{
Six new candidate ultracompact X-ray binaries
}

\author{
J. J. M. in 't Zand ${ }^{1,2}$, P. G. Jonker ${ }^{1,2,3}$, and C. B. Markwardt ${ }^{4,5}$ \\ 1 SRON Netherlands Institute for Space Research, Sorbonnelaan 2, 3584 CA Utrecht, The Netherlands \\ e-mail: jeanz@sron.nl \\ 2 Astronomical Institute, Utrecht University, PO Box 80000, 3508 TA Utrecht, The Netherlands \\ 3 Harvard-Smithsonian Center for Astrophysics, 60 Garden Street, Cambridge, MA 02138, USA \\ 4 Department of Astronomy, University of Maryland, College Park, MD 20742, USA \\ 5 Astroparticle Physics Laboratory, Mail Code 661, NASA Goddard Space Flight Center, Greenbelt, MD 20771, USA \\ Received 1 November 2006 / Accepted 8 January 2007
}

\section{ABSTRACT}

\begin{abstract}
Ultracompact X-ray binaries (UCXBs) appear able to sustain accretion onto the compact accretor at rates lower than in wider X-ray binaries. This may be understood by the smaller accretion disks in UCXBs: a lower X-ray luminosity suffices to keep a disk completely ionized through irradiation and, thus, keep the viscosity at a sufficiently high level to allow effective transport of matter to the compact object. We employ this distinguishing factor on data from RXTE and BeppoSAX to identify six new candidate UCXBs, thus increasing the population by one quarter. The candidates are drawn from the population of persistently accreting and type-I X-ray bursting lowmass X-ray binaries. The X-ray bursts establish the low-mass X-ray binary nature and provide a handle on the accretion rate. We find that the low accretion rates are supported by the long burst recurrence times and the hard X-ray spectra of the persistent emission as derived from the 2 nd INTEGRAL catalog of soft $\gamma$-ray sources. We discuss the peculiar light curves of some new UCXB candidates.
\end{abstract}

Key words. X-rays: binaries - X-rays: bursts - accretion, accretion disks

\section{Introduction}

Ultracompact X-ray binaries (UCXBs) are binaries with orbital periods shorter than $P_{\text {orb }} \approx 1 \mathrm{~h}$ in which a neutron star or black hole accretes matter from a companion star. They are a subset of the low-mass X-ray binaries (LMXBs). The short orbital period implies such a small Roche lobe that the donor star must be hydrogen-poor (Nelson et al. 1986; Savonije et al. 1986). This has 2 interesting implications: 1) they present interesting laboratories to study accretion and thermonuclear burning on neutron star surfaces under hydrogen-poor conditions; and 2) the donor's low-mass (i.e., a few hundredths of a solar mass) inner core is stripped from its outer layers yielding an unimpeded view of the ashes of the stellar nuclear burning which can be studied when dumped on a companion neutron star or black hole (e.g., Deloye $\&$ Bildsten 2003).

Finding UCXBs is difficult, because measuring $P_{\text {orb }}$ is difficult in LMXBs. Only for eight LMXBs has $P_{\text {orb }}$ been measured with certainty to be in the ultracompact regime, see Table 1 . We call these certain UCXBs. The shortest is $11 \mathrm{~min}$, two systems reside at 21 (or 13) and $23 \mathrm{~min}$ and the remaining five systems are between 40 and $50 \mathrm{~min}$. Furthermore, there are four LMXBs for which tentative measurements of $P_{\text {orb }}$ exist. The three UCXBs in globular clusters are all among the shortest binaries. UCXBs are five times more common in globular clusters (where $50 \%$ of all measured $P_{\text {orb }}$ 's are less than $1 \mathrm{~h}$ ) than in the Galactic field (Deutsch et al. 2000; Verbunt \& Lewin 2006). These differences must be related to different evolutionary scenarios between cluster and field systems. The large probability of stellar encounters in globular clusters is an appealing explanation (Fabian et al. 1975).

Identification of UCXBs is most directly done through measuring $P_{\text {orb }}$. There are three methods for this measurement: 1) through timing of Doppler-delayed pulses if the accretor is a pulsar (four detections of certain UCXBs resulted from this, most notably three transient accretion-powered millisecond pulsars); 2) through measuring periodic eclipses, dips or modulations of X-rays if the inclination angle is high enough (two detections); and 3) through measurements of periodic optical modulations (two detections) resulting possibly either from heating one side of the donor by X-ray irradiation or from the superhump phenomenon that is predicted for mass ratios far from 1 (like expected in many UCXBs).

There are two indirect methods to identify UCXBs without measuring $P_{\text {orb }}$. Both depend on the notion that in an UCXB the accretion disk must be relatively small. The first indirect method concerns the ratio of optical to X-ray flux. At the same X-ray flux, $M_{V}$ is about 4 mag fainter for UCXBs than for nonultracompact LMXBs (van Paradijs \& McClintock 1994). Seven UCXBs without measured $P_{\text {orb }}$ 's have so far been identified following this method (e.g., Juett et al. 2001). The second indirect method concerns the critical accretion rate below which a system becomes transient and is employed in this paper to identify six new UCXBs. We present the principle of the method in Sect. 2, the tools in Sect. 3, the data in Sect. 4, the results in Sect. 5, corroborative evidence in Sect. 6 and a discussion of the results in Sect. 7.

We note that there are additional promising diagnostics for a UCXB nature and they are all based on spectral data. The most direct method involves measuring the composition of the donor through spectral lines and edges in the optical accretion disk spectrum (e.g., Nelemans et al. 2004; Werner et al. 2006), in particular the absence of hydrogen lines. X-ray spectroscopy was initially also promising: Juett et al. (2001) and Juett \& Chakrabarty (2003) interpreted unusually high Ne/O abundance ratios as due to a UCXB nature. However, later these ratios turned out to be variable, thus weakening this interpretation (Juett \& Chakrabarty 2005). Finally, Sidoli et al. (2001) made 
Table 1. List of 27 (candidate) UCXBs (adapted from Nelemans \& Jonker 2006a), including 6 cases proposed here on the basis of very low $L_{\mathrm{X}}$. We leave out cases identified through the diagnostic of the X-ray continuum parameter values (Sidoli et al. 2001), for instance EXO 1745-248 in Terzan 5 (Heinke et al. 2003), because that diagnostic is not always consistent with others (e.g., Verbunt \& Lewin 2006).

\begin{tabular}{|c|c|c|c|c|}
\hline Name & (1) & $(2)$ & (3) & $\begin{array}{l}P_{\text {orb }} \\
(\mathrm{min})\end{array}$ \\
\hline \multicolumn{5}{|c|}{ certain $U C X B s$} \\
\hline XTE J0929-314 & $\mathrm{pp}$ & $\mathrm{T}$ & $\bar{M}$ & $44^{a}$ \\
\hline 4U 1626-67 & $\mathrm{pp}$ & $\mathrm{P}$ & $\mathrm{P}$ & $42^{b}$ \\
\hline XTE J1751-305 & $\mathrm{pp}$ & $\mathrm{T}$ & $\mathrm{M}$ & $42^{c}$ \\
\hline XTE J1807-294 & $\mathrm{pp}$ & $\mathrm{T}$ & M & $40^{d}$ \\
\hline 4U 1820-303 (in NGC 6624) & $\mathrm{px}$ & $\mathrm{P}$ & B & $11^{e}$ \\
\hline $4 \mathrm{U} 1850-087$ (in NGC 6712) & po & $\mathrm{P}$ & B & 21 or $13^{f}$ \\
\hline $4 \mathrm{U} 1915-05$ & px & $\mathrm{P}$ & $\mathrm{B}, \mathrm{D}$ & $50^{g}$ \\
\hline M15 X-2 (in M15) & po & $\mathrm{P}$ & $\mathrm{B}$ & $23^{h}$ \\
\hline \multicolumn{5}{|c|}{ candidate UCXBs with tentative orbital periods } \\
\hline $4 \mathrm{U} 0614+091$ & po,r & $\mathrm{P}$ & B & $50^{i}$ \\
\hline 4U 1543-624 & po & $\mathrm{P}$ & & $18^{j}$ \\
\hline H 1825-331 (in NGC 6652) & po & $\mathrm{P}$ & B & $55^{k}$ \\
\hline NGC 6652 B (in NGC 6652) & po & Q & & $44^{k}$ \\
\hline \multicolumn{5}{|c|}{ candidate UCXBs with low optical to X-ray flux } \\
\hline 4U 0513-40 (in NGC 1851) & $\mathrm{r}^{l}$ & $\mathrm{P}$ & $\mathrm{B}$ & \\
\hline 2S 0918-549 & $\mathrm{r}^{l}$ & $\mathrm{P}$ & B & \\
\hline 1A $1246-588$ & $\mathrm{r}^{m}, \mathrm{x}$ & $\mathrm{P}$ & B & \\
\hline 4U 1812-12 & $\mathrm{r}^{m}, \mathrm{X}$ & $\mathrm{P}$ & B & \\
\hline 4U 1822-000 & $\mathrm{r}^{l}$ & $\mathrm{P}$ & & \\
\hline 4U $1905+000$ & $\mathrm{r}^{n}$ & $\mathrm{~T}$ & B & \\
\hline$\omega$ Cen qLMXB & $\mathrm{r}^{o}$ & Q & & \\
\hline \multicolumn{5}{|c|}{ candidate UCXBs based on method here discussed } \\
\hline 1RXS J170854.4-321857 & $\mathrm{x}^{p}$ & $\mathrm{P}$ & $\mathrm{B}$ & \\
\hline SAX J1712.6-3739 & $\mathrm{x}$ & $\mathrm{P}$ & B & \\
\hline 1RXS J171824.2-402934 & $\mathrm{x}^{p}$ & $\mathrm{P}$ & B & \\
\hline 4U 1722-30 (in Terzan 2) & $\mathrm{x}$ & $\mathrm{P}$ & B & \\
\hline 1RXS J172525.2-325717 & $\mathrm{x}$ & $\mathrm{P}$ & B & \\
\hline SLX 1735-269 & $\mathrm{x}$ & $\mathrm{P}$ & B & \\
\hline SLX 1737-282 & $\mathrm{x}$ & $\mathrm{P}$ & B & \\
\hline SLX 1744-299 & $\mathrm{x}$ & $\mathrm{P}$ & $\mathrm{B}$ & \\
\hline
\end{tabular}

(1) Type of argument for ultracompact identification: $\mathrm{r}=L_{\mathrm{x}} / L_{\mathrm{opt}}$ argument, $\mathrm{p}=$ period measurement $(\mathrm{pp}=$ pulsar, $\mathrm{px}=$ dips/eclipse, po = optical modulation), $\mathrm{x}=$ persistent burster with low L; (2) Type of accretion: $\mathrm{P}=$ persistent, $\mathrm{T}=$ transient, $\mathrm{Q}=$ quiescent (never seen to be luminous); (3) Type of source: $\mathrm{P}=$ pulsar, $\mathrm{M}=$ accretion-powered millisecond pulsar, $\mathrm{B}=$ burster, $\mathrm{D}=$ eclipser and/or dipper; ${ }^{a}$ Galloway et al. (2002); ${ }^{b}$ Middleditch et al. (1981); ${ }^{c}$ Markwardt et al. (2002); ${ }^{d}$ Markwardt et al. (2003); ${ }^{e}$ Stella et al. (1987); ${ }^{f}$ Homer et al. (1996); ${ }^{g}$ White \& Swank (1982); ${ }^{h}$ Dieball et al. (2005); ${ }^{i}$ O'Brien et al. (2005); ${ }^{j}$ Wang \& Chakrabarty (2004); ${ }^{k}$ Heinke et al. (2001); ${ }^{l}$ Juett et al. (2001); ${ }^{m}$ Bassa et al. (2006); ${ }^{n}$ Jonker et al. (2006); ${ }^{o}$ Haggard et al. (2004); ${ }^{p}$ in 't Zand et al. (2005a).

a comparison of the X-ray continua spectra of bright globular cluster LMXBs and noted a dichotemy between ultracompact and non-ultracompact cases; the parameters of the disk black body components in ultracompact cases appear to be physically more realistic and consistent with Comptonization components than those in non-ultracompact cases.

\section{Principle: a critical mass transfer rate below which accretion becomes transient}

Cataclysmic variables (CVs; Smak 1983; Osaki 1996) and LMXBs (White et al. 1983) remain in a persistently accreting state if the mass transfer rate from the donor star is above a certain critical value $\dot{M}_{\text {crit }}$. The disk instability model (e.g.,
Osaki 1974; Hoshi 1979; Osaki 1996; Lasota 2001) provides a natural explanation for this. Below the critical value the disk is unstable and steady accretion is impossible. Since $\dot{M}_{\text {crit }}$ is strongly increasing with radius it is its value at the outer disk radius that determines its stability. Therefore, the value of $\dot{M}_{\text {crit }}$ is a strong function of the orbital period and mass (Smak 1983). Furthermore, there is a distinction between CVs and LMXBs because X-ray irradiation is an important effect in the ionization balance of LMXB accretion disks while it is not in CVs (van Paradijs 1996).

Evaluating $\dot{M}_{\text {crit }}$ is difficult, because of the various uncertainties in the nature of the viscosity and the geometry of the irradiation (related to the questions whether the source is point like or extended with respect to the disk and whether the disk is warped). Dubus et al. (1999) and Lasota (2001) derive ${ }^{1}$

$\dot{M}_{\text {crit }}=5.3 \times 10^{-11} C \quad M_{1}^{0.3} P_{\text {orb }}^{1.4} \quad M_{\odot} \mathrm{yr}^{-1}$

with accretor mass $M_{1}$ (in $M_{\odot}$ ) and orbital period $P_{\text {orb }}$ (in h). $C \approx 1$ represents uncertainty ${ }^{2}$. For $P_{\text {orb }}<1 \mathrm{~h}$ and $M_{1}=1.4 M_{\odot}$, $\dot{M}_{\text {crit }} \lesssim 6 \times 10^{-11} M_{\odot} \mathrm{yr}^{-1}$. This value is for solar composition. Menou et al. (2002) calculated values for other compositions, although for the non-irradiated case. For $\mathrm{C} / \mathrm{O}$ disks the results are equal to the solar composition case within a few tens of percents. For pure He or $\mathrm{O}$ disks, the value is 6 times larger. Therefore, if we naively apply this factor to the irradiated case, $\dot{M}_{\text {crit }}$ is approximately $1 \%$ or less of the Eddington limit for a hydrogenpoor/helium-rich photosphere of a bursting $1.4 M_{\odot}$ neutron star. Herein lies the principle of our method: a bursting LMXB may be tentatively identified as a UCXB if it is persistent at accretion luminosities below $\approx 1 \%$ of Eddington.

It is difficult to measure $\dot{M}$, because the uncertainties are large in translating observed flux to bolometric luminosity (i.e., uncertainties in bolometric and anisotropy corrections) and in translating bolometric luminosity to mass accretion rate (i.e., uncertainty in radiation efficiency). Combined with the uncertainty in $\dot{M}_{\text {crit }}$ this makes a sensible test between both accretion rates impossible. Nevertheless, there is merit in the principle. It turns out that if we rank persistently accreting LMXBs according to their ratios of estimated bolometric flux to Eddington flux, all UCXBs with known (tentative) $P_{\text {orb }}$ 's except one populate the lowest $\dot{M}$ regime.

\section{Tool: the X-ray burst phenomenon in UCXBs}

We apply the principle just described to those persistently accreting LMXBs that exhibit type-I X-ray bursts (or shortly, "X-ray bursts") which provides an easy estimate of the Eddington flux. X-ray bursts result from thermonuclear flashes of hydrogen or helium in the freshly accreted layers of neutron stars (for reviews, see e.g. Lewin et al. 1993; and Strohmayer \& Bildsten 2006). For a few seconds to minutes the flashes heat up the atmosphere to few-keV temperatures and the resulting X-ray spectra are dominated by easy-to-analyze black body shapes. The peak fluxes are close to the Eddington limit and for many luminous bursts even equal to it.

Fortunately, many UCXBs exhibit X-ray bursts, although it is not really understood why. Four of the eight certain UCXBs exhibit type-I X-ray bursts, as do seven of the eleven candidate

1 This is Eq. (36) in Lasota (2001) after correcting the numerator of Eq. (34), which was used in deriving Eq. (36), from $M_{2}$ to $M_{1}$ (Lasota, priv. comm.).

${ }^{2}$ We employ another expression for $C$ than Dubus et al. (1999) and Lasota (2001). 


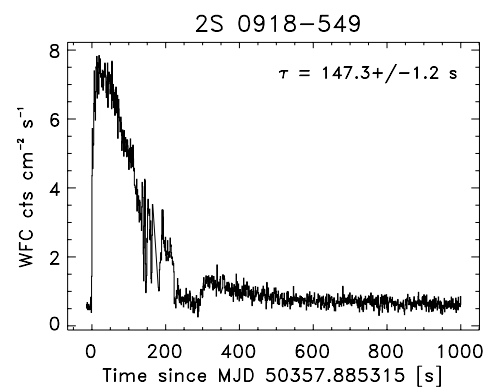

SLX 1735-269

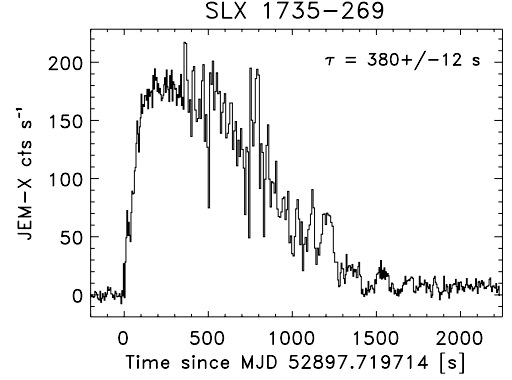

A1246-588

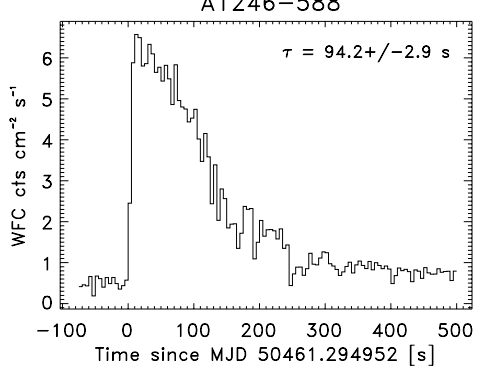

SLX 1737-282

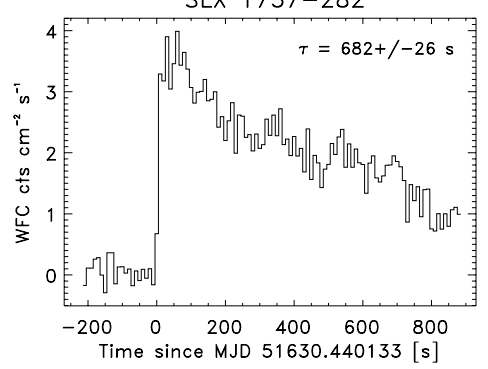

RX J1718.4-4029

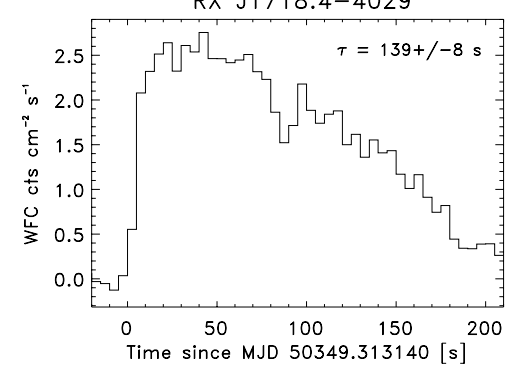

M15 X-2

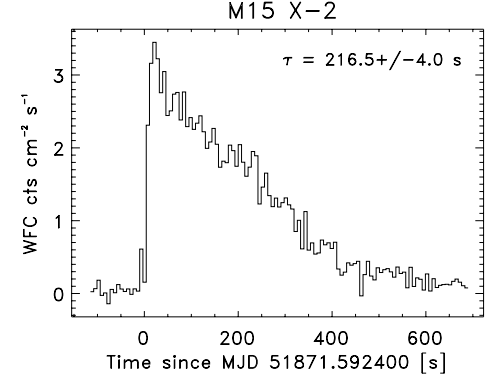

Fig. 1. A compilation of $2-30 \mathrm{keV}$ time profiles of the longest bursts from 5 (candidate) UCXB candidates with BeppoSAX/WFC and 1 with INTEGRAL/JEM-X (Molkov et al. 2005). Indicated are the e-folding decay times resulting from a fit to the data beyond the peak and excluding clear deviations from an exponential as for instance in $2 \mathrm{~S}$ 0918-549. All these bursts start with a radius-expansion phase.

UCXBs. Since the hydrogen abundance in UCXBs is neglible, the bursts must result from flashes of helium being accreted from the companion star. A conclusive confirmation of this argument would be the detection of helium in the optical spectrum. Within the group of UCXBs that burst and have (tentative) $P_{\text {orb }}$ values, this confirmation is there for only one case (XB 1916-05; Nelemans et al. 2006b) showing a He-II emission line at $4690 \AA$, albeit comparatively weak, while it is significantly absent in another (4U 0614+091; Nelemans et al. 2004; and Werner et al. 2006). The remaining cases lack good enough optical spectra for definite verification although $\mathrm{He}$ lines should have been detected in some cases if they would have had similar equivalent widths as in wide-orbit LMXBs. The lack of He lines is one of the reasons why the presence of X-ray bursts is not understood (e.g., Nelemans et al. 2006b). Disregarding that, we use the presence to our advantage.

Often the X-ray bursts from both certain as well as candidate UCXBs are rather long. They sometimes are the longest after the superbursts (up to $40 \mathrm{~min}$, e.g. in 't Zand et al. 2005b; for a few examples see Fig. 1). Cumming et al. (2006), therefore, coined the term "intermediately long X-ray bursts". In general, burst duration is determined by the thickness and hydrogen abundance of the flash layer immediately prior to the flash. Both of these depend foremost on the mass accretion rate. Most bursts are short with a duration of approximately $10 \mathrm{~s}$ and they occur in a very specific accretion rate regime (Fujimoto et al. 1981; Bildsten 1998). The reason that there are so many of them is that many LMXB reside in this accretion regime and the associated burst recurrence time is short (i.e., of order a few hours). The relevant accretion regime is roughly between 1 and 10\% of Eddington (for details, see Bildsten 1998, and references therein). The conditions in this regime are such that between flashes the freshly accreted hydrogen is stably burned at a rate as it is accreted, thus developing a pure helium layer. When the helium layer is thick enough, the pressure at the bottom surpasses ignition conditions for the runaway $3 \alpha$ process. The flash ends within $1 \mathrm{~s}$. The burst duration is a direct measure of the layer cooling time (between a few seconds and a few tens of seconds) and thus depends on the layer thickness. Outside this accretion regime bursts are longer. If the accreted matter is hydrogen rich, this is due to slow beta decay of the products of rapid proton capture by the ashes of the initial helium or hydrogen flash, prolonging the nuclear energy generation by a few minutes. If the accreted matter is hydrogen poor, a long burst is due a low layer temperature if the long-term average accretion rate is so low that heating by pycnonuclear reactions in the crust is significantly reduced (see in 't Zand et al. 2005a; and Cumming et al. 2006) ${ }^{3}$. Only long bursts of the latter kind can occur in UCXBs.

It is expected that X-ray bursts of the latter kind easily reach the Eddington limit resulting in photospheric radius expansion (PRE), because the triple- $\alpha$ reaction rates are fast and the amount of helium large. Also, the PRE phase may last long because of the large helium layer thickness (of order 1 min; cf., Cumming et al. 2006). The long mixed hydrogen/helium flashes do not last longer than a few hundred seconds and may have irregularly shaped decay phases due to the various waiting points in the rpprocess reaction chain (e.g., Schatz et al. 2001; Woosley et al. 2004). The characteristics of the various long X-ray bursts suggest that a burst with a duration in excess of a few minutes and exhibiting long PRE is a unique diagnostic of a UCXB. This appears to be consistent with the fact that such bursts have never, as far as we know, been detected from non-ultracompact systems.

Nevertheless, we do not employ such bursts as a prime diagnostic. Recent modeling of the effect of sedimentation on burst ignition by Peng et al. (2006) shows that for certain low mass accretion rates (below $1 \%$ of the Eddington limit) of hydrogenrich material (ergo, in a non-ultracompact binary) there may exist a burst regime with flashes of pure hydrogen in a layer that is too thin to ignite helium. These flashes produce helium through the CNO cycle. A thick helium layer grows which may result in a similarly long burst as described above for a hydrogen-poor ultracompact case at low accretion rate. There appear two issues concerning the viability of this burst regime: 1) as Peng et al. (2006) discuss, for mass accretion rates of order $1 \%$ of the

\footnotetext{
3 Crustal heating reacts to mass accretion rate on time scales of months and, thus, is independent of short term variability.
} 
Eddington limit the pure helium layer would grow to $10^{11} \mathrm{~g} \mathrm{~cm}^{-2}$ before ignition would occur which would result in burst durations comparable to those of short superbursts which has never been observed; 2) as we have discussed in Sect. 2, the low accretion rates needed may not be possible in non-ultracompact cases because the disk instability would turn off the accretion. Resolution of these issues is desirable but difficult because of the nature of the objects involved: quite faint objects with predicted superburst-like flares every few years (note that these are inconsistent with "burst-only" sources; see Cornelisse et al. 2002a,b).

\section{Data}

\subsection{Average persistent fluxes of persistent $X$-ray bursters}

We collected data on all $40 \mathrm{X}$-ray bursters which, to the best of our knowledge, are currently active and have been so for longer than five years, see Table 2. It is fair to assume that the accretion in these systems is persistent because the viscous timescales in the accretion disk are thought to be only a few months (e.g., Lasota 2001 and references therein). To estimate the $a v$ erage bolometric flux, we employed flux data from two longterm monitoring observations. The flux in persistent LMXBs is known to fluctuate up to an order of magnitude on time scales of up to hundreds of days. Therefore, a one-time flux measurement may not be a good representation of the mass transfer rate from the companion star. Long-term monitoring observations are crucial. We appealed to 1) public data from the Rossi X-ray Timing Explorer (RXTE) All-Sky Monitor (ASM) which has been gathering data on most of these sources since January 1996 (Levine et al. 1996; Wen et al. 2006) in the $2-12 \mathrm{keV}$ band each day for roughly 10 months of the year at a sensitivity of about $5 \times 10^{-10} \mathrm{erg} \mathrm{cm}^{-2} \mathrm{~s}^{-1}$ per day. Sources which are not included are either too faint to be detectable, or too near a bright source to allow accurate flux measurements; 2) the RXTE Proportional Counter Array (PCA) Galactic Bulge monitoring program which has been running on the inner $16^{\circ}$ of the bulge since February 1999 and on the inner 52 ${ }^{\circ}$ since May 2004 (Swank \& Markwardt 2001; Markwardt 2006), measuring the flux of every source twice a week in the $2-12 \mathrm{keV}$ band for 10 months of each year at a sensitivity of about $10^{-11} \mathrm{erg} \mathrm{cm}^{-2} \mathrm{~s}^{-1}$. There are four sources (1RXS J171824.2-402934, SLX 1744-299, SLX 1744-300 and M15 X-2) for which there are no accurate ASM nor PCA measurements due to faintness or source confusion and we use results from targeted more sensitive observations. For these, we lack long enough exposures to assess a good long-term average, although it should be said that they, apart from during X-ray bursts, have never been seen in a bright state.

In order to derive from the observed photon flux an estimate of the bolometric energy flux, the absolute calibrations provided for the two data sets are employed: the Crab source yields $75 \mathrm{ASM} \mathrm{c} \mathrm{s}^{-1}$ and $11350 \mathrm{PCA} \mathrm{c} \mathrm{s}^{-1}$ (normalized to 5 Proportional Counter Units). The Crab spectrum is a power law with a photon index of 2.1 and $N_{\mathrm{H}}=4 \times 10^{21} \mathrm{~cm}^{-2}$ (Kirsch et al. 2005). If in fact the photon index of the actual source differs by 1 or $N_{\mathrm{H}}$ is up to 10 times larger, the 2-10 or $2-12 \mathrm{keV}$ energy flux differs by at most $30 \%$. We assume this to be the uncertainty in translating the $2-10 \mathrm{keV}$ observed photon flux to $2-10 \mathrm{keV}$ intrinsic energy flux. The next step is the bolometric correction. The literature on broad-band spectra of LMXBs frequently provides absorbed fluxes in $2-10 \mathrm{keV}$ as well as unabsorbed $0.1-100 \mathrm{keV}$ fluxes. We browsed the literature to obtain characteristics values for the flux ratio and find
$2.9 \pm 1.4$ to be a fair representation. Thus, we have a conversion of $1 \mathrm{ASM} \mathrm{c} \mathrm{s}^{-1}$ to $7.7 \times 10^{-10} \mathrm{erg} \mathrm{cm}^{-2} \mathrm{~s}^{-1}$ and $1 \mathrm{PCA} \mathrm{c} \mathrm{s}^{-1}$ to $5.1 \times 10^{-12} \mathrm{erg} \mathrm{cm}^{-2} \mathrm{~s}^{-1}$, with a typical error of a factor of 2 . It is not really worthwhile to try to assess the spectrum individually per source from archival data and derive conversion factors from that because the relevant data are mostly snapshots that are not representative of the average behavior and because $N_{\mathrm{H}}$ measurements usually suffer from such large systematic errors that the correction for absorption, important below a few $\mathrm{keV}$, is quite inaccurate.

An additional correction is necessary for the inclination angle: if the disk is viewed near edge-on, the observable flux reduces considerably and needs to be corrected by a factor of $\xi_{\mathrm{p}}^{-1}=2|\cos i|$ (Lapidus \& Sunyaev 1985; Fujimoto 1988). $\xi_{\mathrm{p}}$ ranges from 0.5 for $i=0^{\circ}$ to $>2$ for $i>75^{\circ}$. For $i \gtrsim 85^{\circ}$ the accretion disk probably blocks the view to the NS and no bursts are observable. Without knowledge of $i, \xi_{\mathrm{p}}$ probably ranges between 0.5 and 2 for bursters. An additional uncertainty is introduced by the efficiency factor $\eta$ with which gravitational energy is transformed to radiation and how representative the flux is for the mass accretion rate. Combining all uncertainties probably adds up to a factor of 3 (i.e., the quadratic sum of 3 factors of 2).

Table 2 provides the average raw photon count rates for ASM and PCA, the extrapolated flux values and a comparison between both values if they are both present (i.e., a few sources are not in the ASM catalog, and half are not covered in the PCA bulge scan program). Only 3 out of 19 comparisons deviate by more than a factor of two. These are also the 3 sources with the lowest fluxes. This probably results from unaccounted-for bias levels in the ASM fluxes. For a constant bias level of $0.3 \mathrm{ASM} \mathrm{c} \mathrm{s}^{-1}$, which is a reasonable number for short angular distances to the Galactic center, the ASM/PCA ratios would remain with a factor of 2 from the value 1 .

\subsection{Eddington fluxes}

The highest bolometric burst peak flux observed for any source in the history of X-ray astronomy provides a lower limit to the Eddington flux for that source. Fortunately, these maxima often apply to bursts which experienced photospheric radius expansion (PRE) and the peak flux is actually equal to the Eddington flux. We searched through the literature to find the highest peak fluxes. For one case we determined the burst peak flux ourselves from BeppoSAX-WFC data, see Fig. 2. The results are listed in Table 2. Fifteen sources did not exhibit any unambiguous PRE bursts.

For canonical neutron stars with a mass of $1.4 M_{\odot}$ and a radius of $10 \mathrm{~km}$, the Eddington limit is $2.0 \times 10^{38} \mathrm{erg} \mathrm{s}^{-1}$ for a hydrogen-rich photosphere and $3.5 \times 10^{38} \mathrm{erg} \mathrm{s}^{-1}$ for a hydrogen-poor photosphere (if the radius expansion is small with respect to the NS radius, a relativistic correction of a factor of $\sqrt{1-2 G M / R c^{2}}$ needs to be applied to these values which equals 0.76 for a canonical NS). Like the persistent flux, the burst peak flux also needs to be corrected for inclination angle. Lapidus \& Sunyaev (1985) and Fujimoto (1988) derive a correction factor of $\xi_{\mathrm{b}}^{-1}=0.5+|\cos i|$. If no orbital modulation is seen in the flux, $i$ may be presumed to be smaller than $70^{\circ}$ and $\xi_{\mathrm{p}}$ lies between 0.84 and 1.5 . Thus, this introduces an uncertainty of about $30 \%$. 
Table 2. 40 persistent X-ray bursters, in order of average to Eddington flux ratio. The division lines are at 2 and $10 \%$.

\begin{tabular}{|c|c|c|c|c|c|c|c|c|c|c|c|}
\hline Source name & $\overline{\mathrm{ASM}}$ & (2) & PCA & (2) & $\begin{array}{l}\text { ASM/ } \\
\text { PCA }\end{array}$ & Other & $\begin{array}{l}\text { Burst } \\
\text { peak } \\
\text { flux } \\
(2,4)\end{array}$ & $\begin{array}{l}\text { Ratio } \\
\% \\
\text { Edd. } \\
(5)\end{array}$ & $\begin{array}{c}\text { Burst rec. } \\
\text { time (h) } \\
(6)\end{array}$ & $\begin{array}{c}\text { Previously } \\
\text { identified } \\
\text { UCXB? }\end{array}$ & $\begin{array}{l}P_{\text {orb }} \\
\text { (h) } \\
\text { (7) }\end{array}$ \\
\hline 1RXS J171824.2(8) & $0.402(9)$ & 3.1 & - & - & & $0.1^{a}$ & $390^{b}$ & 0.03 & $438-8254$ & & \\
\hline SLX 1737-282 & - & - & $47.8(7)$ & 2.4 & & $2.3^{c}$ & $\overline{600}^{c}$ & 0.4 & $412-7778$ & & \\
\hline 2S 0918-549 & $0.576(5)$ & 4.5 & - & - & & $6.0^{d}$ & ${\overline{1000^{d}}}^{d}$ & 0.5 & $202-853$ & cand. UCXB & \\
\hline 1A $1246-588$ & $0.618(6)$ & 4.8 & - & - & & & 900 & 0.5 & $278 \pm 139$ & cand. UCXB & \\
\hline SAX J1712.6-3739 & $0.868(9)$ & 6.7 & $50.7(7)$ & 2.6 & 2.58 & & $\overline{510^{e}}$ & 0.5 & $345-6507$ & & \\
\hline 4U 1812-12 & $1.328(7)$ & 10.3 & $166.1(8)$ & 8.5 & 1.21 & $10.9^{f}$ & $\overline{1600}$ & 0.5 & $80.2 \pm 18.9$ & cand. UCXB & \\
\hline 4U 1850-087 & $0.606(5)$ & 4.7 & $52.1(2)$ & 2.7 & 1.74 & $11.9^{g}$ & $\overline{\underline{600}^{h}}$ & 0.5 & $>1584$ & $\mathrm{UCXB}$ & 0.3 \\
\hline 1RXS J172525.2-3257 & $717 \quad-$ & - & $24.9(2)$ & 1.3 & & & $230^{a c}$ & 0.6 & & & \\
\hline 4U 0614+091 & $3.111(5)$ & 24.1 & - & - & & & $\underline{3000}^{i}$ & 0.8 & $168-3175$ & cand. UCXB & 0.8 \\
\hline SLX 1735-269 & $1.25(1)$ & 9.7 & $107.0(3)$ & 5.5 & 1.76 & & $\overline{577}^{j}$ & 1.0 & $387-7301$ & & \\
\hline EXO 0748-676 & $0.668(5)$ & 5.2 & - & - & & & $\overline{520}^{k}$ & 1.0 & $5.1 \pm 0.4$ & & 3.8 \\
\hline 4U 1915-05 & $1.001(5)$ & 7.7 & - & - & & & $\overline{646}^{l}$ & 1.1 & $31 \pm 11$ & $\mathrm{UCXB}$ & 0.8 \\
\hline H 1825-331 & $0.665(9)$ & 5.1 & $70.5(5)$ & 3.6 & 1.42 & $4.3^{m}$ & $297^{n}$ & 1.2 & $27.8 \pm 7.4$ & cand. UCXB & \\
\hline M15 X-2 ${ }^{(9)}$ & $0.565(3)$ & 4.4 & - & - & & $3.8^{g}$ & $\overline{375}^{p}$ & 1.2 & 37-984 & UCXB & 0.4 \\
\hline XTE J1710-281 & $0.425(12)$ & 3.3 & $23.04(5)$ & 1.2 & 2.75 & & $\overline{9 \underline{9}^{n}}$ & 1.3 & & & 3.9 \\
\hline 1RXS J170854.4-3218 & $857 \quad-$ & - & - & - & & $2.4^{a}$ & $1 \overline{54}^{a}$ & 1.5 & 101-1904 & & \\
\hline $4 \mathrm{U} 1722-30$ & $2.06(1)$ & 15.9 & $246.4(4)$ & 12.6 & 1.26 & $18^{g}$ & $\overline{708}^{\circ}$ & 1.8 & $57 \pm 12$ & & \\
\hline 4U 0513-40 & $0.411(4)$ & 3.2 & - & - & & & $\overline{170}^{n}$ & 1.9 & $49 \pm 14$ & cand. UCXB & \\
\hline SLX 1744-299 ${ }^{(10)}$ & $0.989(8)$ & 7.7 & $163(5)$ & 8.3 & 0.92 & $12^{r}$ & $420^{q}$ & 1.9 & $188-793$ & & \\
\hline 4U 1746-37 & $2.306(8)$ & 17.8 & $318.6(5)$ & 16.3 & 1.09 & & $\underline{630}^{n}$ & 2.6 & & & 5.7 \\
\hline A $1742-294$ & - & - & 213.2(19) & 10.9 & & & $\overline{401}^{n}$ & 2.7 & $6.1 \pm 0.4$ & & \\
\hline 4U 1702-429 & $3.191(8)$ & 24.7 & $429.0(11)$ & 21.9 & 1.13 & & $\overline{810}^{n}$ & 2.7 & $11.4 \pm 1.0$ & & \\
\hline XTE J1759-220 & $0.556(10)$ & 4.3 & $31.45(8)$ & 1.6 & 2.69 & & $\overline{51^{n}}$ & 3.1 & & & $1-3$ \\
\hline SLX $1744-300^{(10)}$ & $0.534(4)$ & 4.1 & $88(3)$ & 4.5 & 0.92 & $6^{r}$ & $190^{n}$ & 3.2 & $24.7 \pm 6.7$ & & \\
\hline 4U 1323-62 & $0.598(8)$ & 4.6 & - & - & & & $107^{n}$ & 4.3 & $39 \pm 11$ & & 2.9 \\
\hline GX 354-0 & $6.311(8)$ & 48.8 & $1031.3(10)$ & 52.7 & 0.93 & & $\underline{1200}^{s}$ & 4.4 & $3.2 \pm 0.2$ & & \\
\hline GS 1826-24 & $2.535(10)$ & 19.6 & $424.8(1)$ & 21.9 & 0.89 & & $330^{t}$ & 6.6 & $4.6 \pm 0.3$ & & $2.1 ?$ \\
\hline 4U 1636-536 & $10.420(7)$ & 80.6 & - & - & & & $742^{n}$ & 10.9 & $8.9 \pm 1.0$ & & 3.8 \\
\hline 4U 1705-440 & $10.857(9)$ & 84.0 & $1082.9(16)$ & 55.3 & 1.52 & & $\underline{410}^{n}$ & 13.5 & $16.5 \pm 1.9$ & & \\
\hline UW Crb & - & - & - & - & & $0.4^{u}$ & $\overline{2.44}^{u}$ & 16.4 & & & 1.9 \\
\hline 4U 1254-69 & $2.420(5)$ & 18.7 & - & - & & $14^{v}$ & $\underline{110}^{w}$ & 17.0 & $44.9 \pm 8.8$ & & 3.9 \\
\hline GX 3+1 & $21.015(12)$ & 162.5 & 2991.4(12) & 152.9 & 1.06 & & $\overline{690}^{x}$ & 22.2 & $21.4 \pm 2.7$ & & \\
\hline 4U 1820-303 & 19.31(1) & 149.3 & 2754.4(13) & 140.8 & 1.06 & & $\overline{570}^{n}$ & 24.7 & $26.6 \pm 3.8$ & UCXB & 0.2 \\
\hline 4U 1708-40 & $1.910(8)$ & 14.8 & $434.8(11)$ & 22.2 & 0.67 & & $\overline{86^{a} a}$ & 25.8 & & & \\
\hline 4U 1735-44 & 13.234(9) & 102.3 & - & - & & & $358^{n}$ & 28.6 & $29.9 \pm 4.8$ & & 4.6 \\
\hline Ser X-1 & $16.189(6)$ & 125.2 & - & - & & & $293^{n}$ & 42.7 & $75 \pm 29$ & & \\
\hline Cir X-1 & $13.766(10)$ & 106.5 & - & - & & & $204^{z}$ & 52.2 & & & 398 \\
\hline GX 13+1 & $22.788(9)$ & 176.2 & $3757.5(30)$ & 192.0 & 0.92 & & $260^{y}$ & 73.8 & & & \\
\hline Cyg X-2 & $35.682(7)$ & 275.9 & - & - & & & $154^{n}$ & 179.1 & & & 236 \\
\hline GX 17+2 & 44.631(10) & 345.1 & 7457.1(42) & 381.1 & 0.91 & & ${\underline{145^{a b}}}^{a}$ & 262.8 & $105 \pm 29$ & & \\
\hline
\end{tabular}

(1) Average instrument intensity in $\mathrm{c} \mathrm{s}^{-1}$, normalized to 5 PCUs for the PCA; includes data up to June 2006; (2) Estimated bolometric flux in $10^{-10} \mathrm{erg} \mathrm{cm}^{-2} \mathrm{~s}^{-1}$; (3) Single flux measurements in $10^{-10} \mathrm{erg} \mathrm{cm}^{-2} \mathrm{~s}^{-1}$ derived from a broader bandpass with, thus, more accurate bolometric corrections; only values given for faint sources, particularly if they are not covered by the PCA bulge scans; (4) if underlined (with a wave) the flux relates to a (tentative) Eddington-limited case; (5) the persistent to burst peak flux ratio, prioritized following PCA flux, "other" flux (note 3) if the ASM data are flat or near expected bias levels (i.e., 0.1 to $0.5 \mathrm{c} \mathrm{s}^{-1}$ ) depending on the sky position, or the ASM flux; (6) Burst recurrence time from BeppoSAX-WFC archive. Uncertainties and lower limits are for $68 \%$ confidence from Poisson statistics; (7) For some references, see Table 1; (8) Full name: 1RXS J171824.2-402934; (9) The flux of M15 X-2 was scaled from the total M15 flux through the fluxes measured for M15 X-2 and AC 211 by White \& Angelini (2001); (10) SLX 1744-299 and SLX 1744-300 are only 2'.8 apart and cannot be separated by the ASM nor PCA. The ASM and PCA fluxes of both sources were scaled according to a flux 1.0/2.8 ratio following Mori et al. (2005); ${ }^{a}$ in 't Zand et al. (2005a); ${ }^{b}$ Kaptein et al. (2000); ${ }^{c}$ in 't Zand et al. (2002a); ${ }^{d}$ in 't Zand et al. (2005b); ${ }^{e}$ Cocchi et al. (2001); ${ }^{f}$ Barret et al. (2003); ${ }^{g}$ Sidoli et al. (2001); ${ }^{h}$ Hoffman et al. (1980); ${ }^{i}$ Kuulkers et al., in prep.; ${ }^{j}$ Molkov et al. (2005); ${ }^{k}$ Wolff et al. (2005); ${ }^{l}$ Smale et al. (1988); ${ }^{m}$ Parmar et al. (2001); ${ }^{n}$ Galloway et al. (2006); ${ }^{o}$ Molkov et al. (2000); ${ }^{p}$ van Paradijs et al. (1990); ${ }^{q}$ Pavlinsky et al. (1994) and in 't Zand et al. in prep.; ${ }^{r}$ Mori et al. (2005) ${ }^{s}$ Galloway et al. (2003); ${ }^{t}$ Galloway et al. (2004); ${ }^{u}$ Hakala et al. (2005) and Hynes et al. (2004); ${ }^{v}$ Iaria et al. (2001); ${ }^{w}$ in 't Zand et al. (2003). ${ }^{x}$ Kuulkers \& van der Klis (2000); ${ }^{y}$ Matsuba et al. (1995); ${ }^{z}$ Tennant et al. (1986); ${ }^{a a}$ Migliari et al. (2003); ${ }^{a b}$ Kuulkers et al. (2002); ${ }^{a c}$ Brandt et al. (2006).

\subsection{Average mass transfer rates in terms of the Eddington limit}

The burst peak flux represents a measurement of the Eddington limit or a lower limit to that (if no PRE bursts were detected). The persistent flux represents the average mass accretion rate which, for persistent sources, is equal to the mass transfer rate. The ratio thus provides an indication for the upper limit to the mass transfer rate in terms of the Eddington limit, with all the uncertainties mentioned above. The ratio numbers provided in Table 2 have been calculated with the best numbers for persistent 


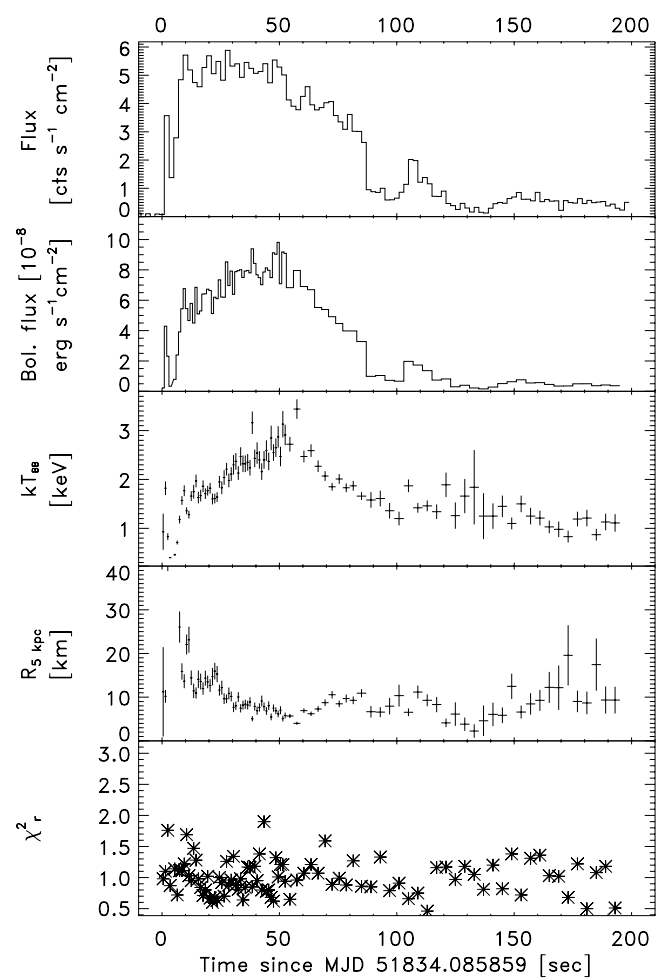

Fig. 2. Time-resolved spectroscopy of burst from 1A 1246-588 obtained with BeppoSAX-WFC.

flux, in other words the PCA bulge scan numbers take preference over the other 2 numbers. If the ASM data show the source to be reasonably constant over the years, the fluxes from independent studies ("other" numbers in table) take preference over the ASM numbers. The rms uncertainty in the flux numbers is expected to be close to a factor of 2 .

Three groups can be distinguished in the ratio distribution: bursters with a mass transfer rate higher than $\sim 10 \%$ of Eddington (13 cases including the highest cases GX 17+2 and Cyg X-2); those with a ratio between $\sim 2$ and $\sim 10 \%$ ( 8 cases); and those with lower ratios (19 cases). Most importantly: all ten persistent and bursting LMXBs that have been identified as ultracompact, except 4U 1820-303, are in the low mass-transfer rate regime.

\subsection{Burst recurrence times}

An interesting parameter is the burst recurrence time since this is dependent on the mass accretion rate on the neutron star: the faster new fuel is provided from the donor, the shorter the burst recurrence time. The recurrence time is not completely inversely proportional to the accretion rate. For accretion rates in excess of 1 to $10 \%$ of the Eddington limit, hydrogen burning is stable and will not give rise to X-ray bursts (e.g., Fujimoto et al. 1981). Thus, there is a sudden change of recurrence time at this threshold value.

For the most common bursters that radiate at about $10 \%$ of the Eddington limit, burst recurrence times are of order a few hours. Going further above and below that the recurrence time increases (e.g., Cornelisse et al. 2003).

Probably the most comprehensive database on burst recurrence time in a time frame coincident with the RXTE data is the BeppoSAX-WFC database, because the exposure time is large (1 to $5 \mathrm{Ms}$ per source). This encompasses $2300 \mathrm{X}$-ray bursts from 54 sources (e.g., in 't Zand et al. 2004). We have used this

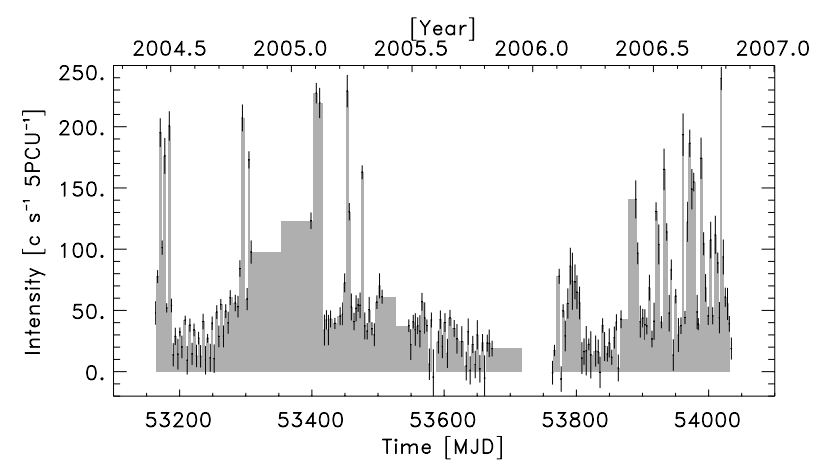

Fig. 3. The 2-12 keV light curve of SAX J1712.6-3739 as measured between May 2004 and November 2006 through the PCA bulge scan program.

database to determine the average burst recurrence time, simply by dividing the total exposure time per source through the number of bursts detected from that source. Some sources were never seen bursting with the WFC because the bursts are probably all below the detection threshold (4U 1708-40, XTE J1710281, 4U 1746-37, XTE J1759-220, UW Crb and Cyg X-2) and we refrain from giving numbers on burst recurrence time. The numbers for the other sources are provided in Table 2. These are based on total exposures for all observations that the sources were sufficiently close to the optical axis that the sensitivity was high enough to detect typical bursts for each source. The minimum required detector area ranges between 5 and $40 \%$ of the optimum on-axis case. Similar percentages of observation time were excluded (i.e., with too far off-axis positions). The $68 \%$ confidence errors are based on Poisson statistics for the counted number of bursts which represents a worst case because bursts commonly do not occur randomly but quasi periodically. The derived burst recurrence times generally follow the trend with accretion rate as described above.

\section{Results: six new candidate UCXBs}

Excluding 2 clear cases of a high inclination angle and, therefore, large cos $i$ correction (EXO 0748-676 and XTE J1710281 ), there are 8 persistent $X$-ray bursters with luminosities below $\approx 2 \%$ of Eddington that are not identified yet as UCXBs, see Table 1. All of these are infrequent bursters (i.e. with recurrence times in excess of a few days), which is consistent with low accretion rates. We propose that these are good candidates for being UCXBs. Two cases were already previously identified on the same grounds (in 't Zand et al. 2005a). The remaining six cases are in order of right ascension:

\subsection{SAX J1712.6-3739}

This source was discovered in 1999 (in 't Zand et al. 1999; Cocchi et al. 1999, 2001) and since then is a persistent source (in 't Zand et al. 2002b), if not earlier: there is a ROSAT All-Sky Survey detection of a source just 0' 6 from the SAX position: 1RXS J171237.1-373834 (in 't Zand et al. 1999). In Fig. 3 is shown the most detailed long-term light curve obtained thus far, with the PCA bulge scan program. During these two years the source is continuously active, apparently in two states: a slowly changing state, and a quicker one. The all-time high in the flux is $230 \mathrm{c} \mathrm{s}^{-1} \mathrm{PCU}^{-1}$ or $1.6 \times 10^{-9} \mathrm{erg} \mathrm{cm}^{-2} \mathrm{~s}^{-1}$ or $3 \%$ of the burstmeasured Eddington limit. 


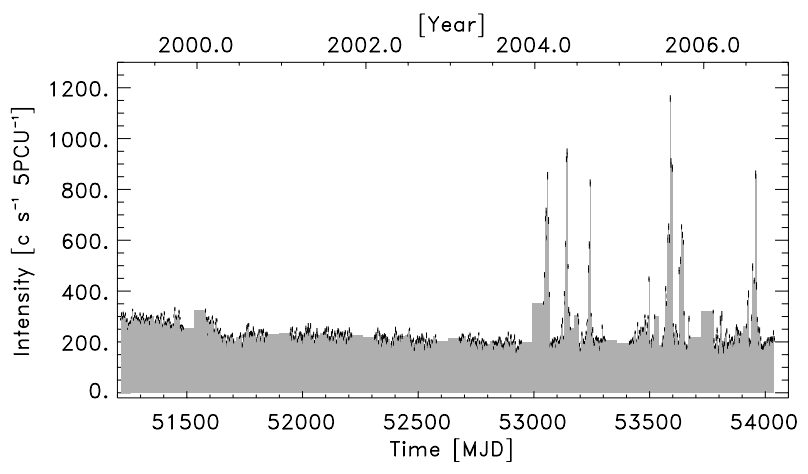

Fig. 4. The $2-12 \mathrm{keV}$ light curve of $4 \mathrm{U} 1722-30$ as measured between February 1999 and November 2006 through the PCA bulge scan program.

In the 4.1 Ms large BeppoSAX-WFC database just a single photospheric radius-expansion burst was detected with a derived distance of 6-8 kpc (Cocchi et al. 2001). Recently, Chelovekov et al. (2006) reported two further burst detections with INTEGRAL-IBIS in 2003-2004. All had the same peak flux.

No optical counterpart has been identified yet within the $13^{\prime \prime}$ $(1 \sigma)$ ROSAT error circle radius. The X-ray absorption column density of $N_{\mathrm{H}}=1.3 \times 10^{22} \mathrm{~cm}^{-2}$ (Cocchi et al. 2001; Dickey \& Lockman 1990) suggests a visual extinction of $A_{\mathrm{V}}=7.3$. Together with the $7 \mathrm{kpc}$ distance this brings the expected visual magnitude to $\approx 26$ for an ultracompact and $\approx 22$ for a nonultracompact binary. Refinement of the error circle through Chandra and optical follow-up may bring confirmation of the UCXB nature.

\section{2. $4 \cup 1722-30$}

This is the bright LMXB in the globular cluster Terzan 2. It was first detected 35 years ago with Uhuru. A 7-yr long X-ray light curve is presented in Fig. 4. It shows the same behavior as for SAX J1712.6-3739 with a slow/faint and quick/bright component, although only in a limited time interval when the slow component is faintest in $2-10 \mathrm{keV}$. Figure 7 shows the light curve zoomed in on four intervals of flaring activity. The shortest typical time scale between flares is $50-100 \mathrm{~d}$.

This system shows regular bursts with an average recurrence time of $2.5 \mathrm{~d}$ according to the BeppoSAX-WFC data archive which contains 24 burst detections (Cocchi et al. 2000b; Kuulkers et al. 2003).

The reddening to Terzan 2 is $E(B-V)=1.57$ (Ortolani et al. 1997), implying a visual extinction of $A_{V}=4.8$ (for $R=3.1$ ). The distance of $9.5 \mathrm{kpc}$ (e.g., Kuulkers et al. 2003) brings the expected apparent visual magnitude to $\approx 24$ for an ultracompact and $\approx 20$ for a non-ultracompact binary. However, the source is located in the core of the cluster where source confusion may be too much of an issue for optical identification, even with a Chandra-determined position.

\section{3. $1 R X S$ J172525.2-325717}

1RXS J172525.2-325717 is a persistent source that is continuously detected in the PCA bulge scans at a low flux, see Fig. 5. There has not been a detailed study of its persistent radiation yet. It is also known as IGR J17254-3257 (see also Stephen et al. 2005; Walter et al. 2004). The only type-I X-ray burst was discovered in 17 February $20043-30 \mathrm{keV}$ data of the JEM-X

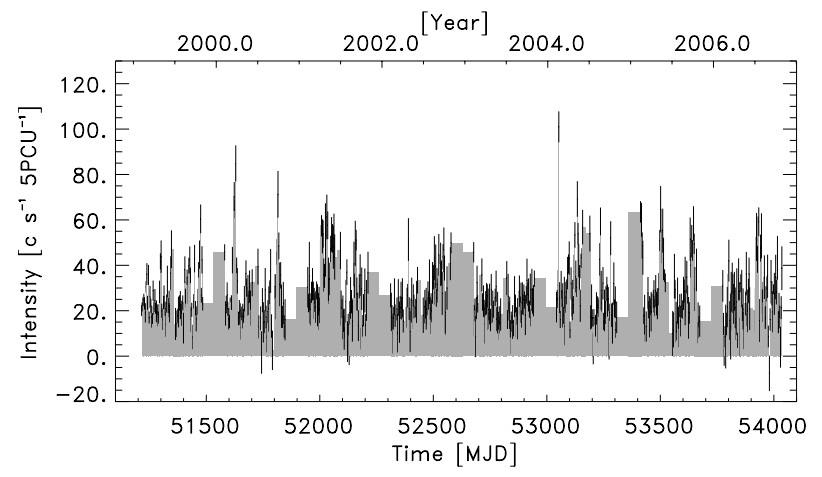

Fig. 5. The 2-12 keV light curve of 1RXS J172525.2-325717 as measured between February 1999 and November 2006 through the PCA bulge scan program.

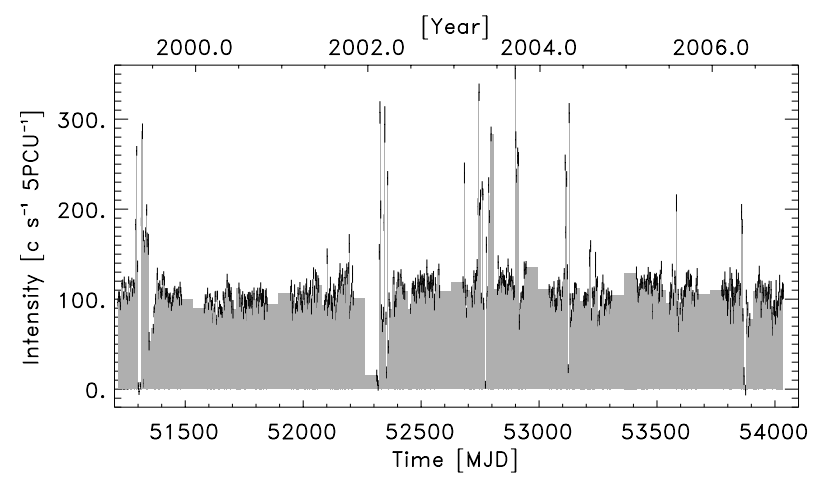

Fig. 6. The 2-12 keV light curve of SLX 1735-269 as measured between February 1999 and November 2006 through the PCA bulge scan program.

camera on INTEGRAL (Brandt et al. 2006). The burst had a rise time of less than $5 \mathrm{~s}$ and an e-folding decay time of $15 \mathrm{~s}$. The peak flux was 0.8 Crab units. For a $2.5 \mathrm{keV}$ spectrum this would translate to roughly a bolometric flux of $2.3 \times 10^{-8} \mathrm{erg} \mathrm{cm}^{-2} \mathrm{~s}^{-1}$.

\subsection{SLX 1735-269}

SLX 1735-369 was discovered in 1985 data taken with the X-ray telecope on Spacelab-2 (Skinner et al. 1987). The source was also detected in 1979 Einstein data by Elvis et al. (1992). It appears to be continuously on. Figure 6 shows the X-ray light curve, again exhibiting the same bimodal behavior as the previous two sources. On top of that, remarkably the flux dips to zero sometimes after a flare, see Fig. 8.

Discovered as an X-ray burster a decade ago through a single short burst with BeppoSAX-WFC (Bazzano et al. 1997), SLX 1735-269 revealed 6 bursts with INTEGRAL (Molkov et al. 2005), one of them being of an extremely energetic and long kind although probably not a carbon-fueled superburst (see Fig. 1). This burst occurred at the start of a brief outburst episode. This prompts the question: is the outburst a result of the energetic burst or vice versa? We investigated the INTEGRAL-IBIS data on the persistent emission at somewhat higher resolution ( $2.5 \mathrm{~h}$ resolution instead of $3 \mathrm{~d}$; see Fig. 9) and find that the transition to the high accretion rate state started $2 \mathrm{~d}$ prior to the burst. Therefore, the long burst probably ignited as a result of an increased accretion rate and not vice versa.

On June 20, 2005, another energetic burst was detected with HETE-2 (Suzuki \& Kawai 2005). Due to absence of PCA data it is not possible to verify another association with an outburst. 

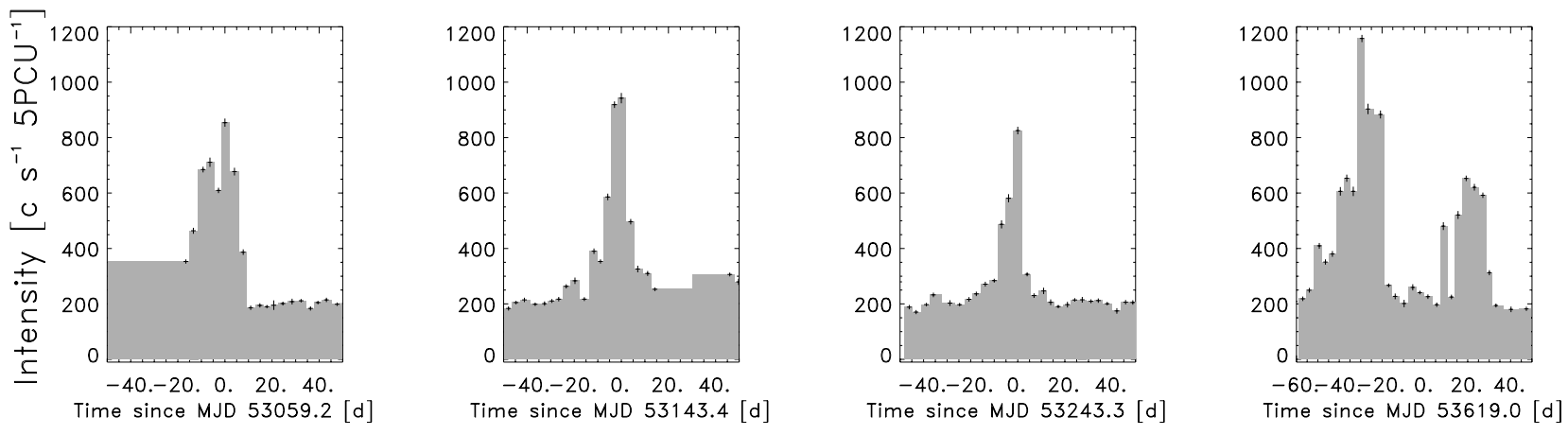

Fig. 7. The lightcurve of $4 \mathrm{U} 1722-30$ (Fig. 4), zoomed in on the flares.
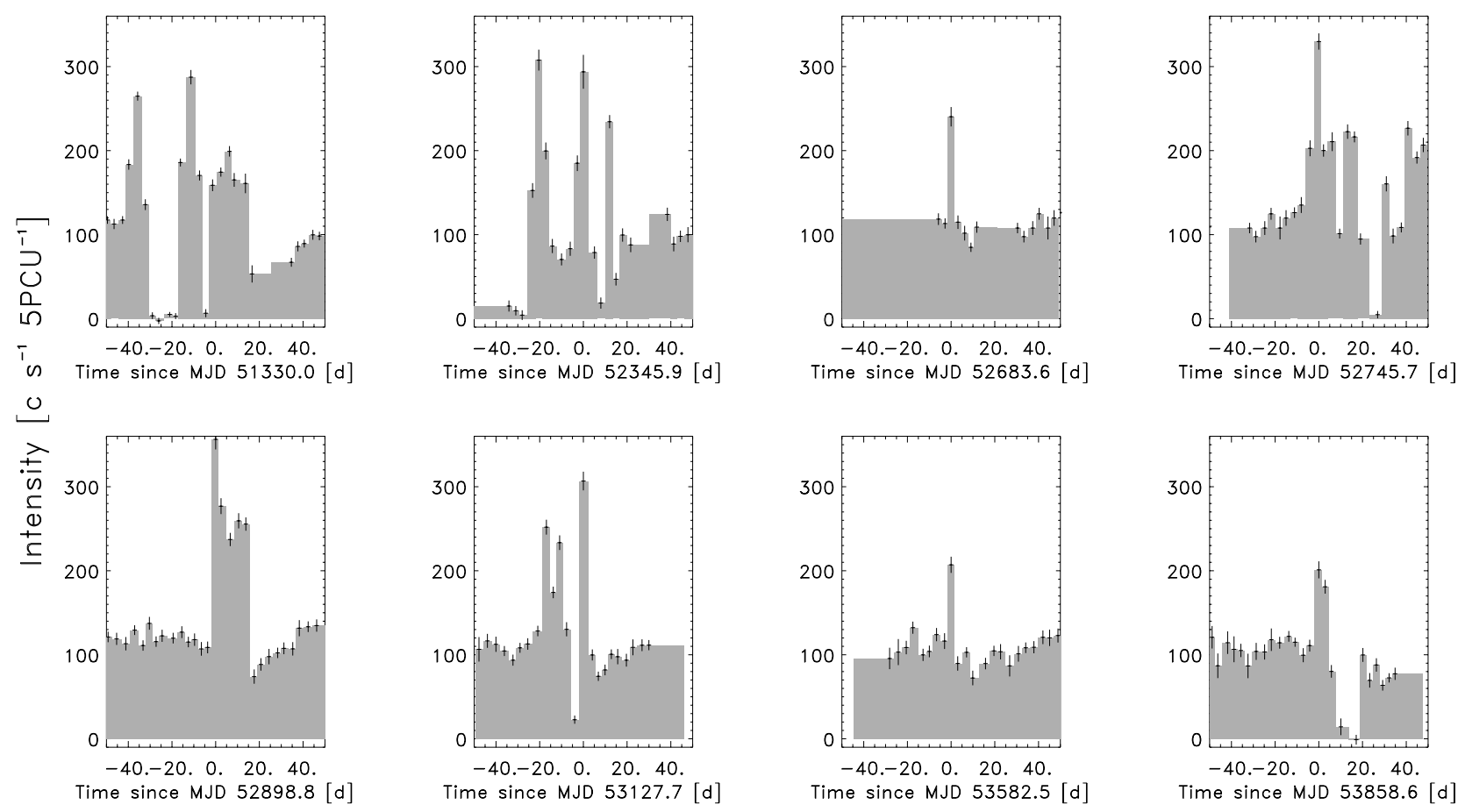

Fig. 8. The lightcurve of SLX 1735-269 (Fig. 6), zoomed in on a 100-d interval around the flares.

The absorption column towards SLX $1735-269$ is $1.7 \times$ $10^{22} \mathrm{~cm}^{-2}$ (Wilson et al. 2003), equivalent to $A_{V}=9.5$. For a distance of $6.5 \mathrm{kpc}$ (Molkov et al. 2005) the expected visual magntitude is $\approx 27$ for an ultracompact and $\approx 23$ for a non-ultracompact binary. Deep optical follow-up of the Chandra position (Wilson et al. 2003) may bring confirmation of the UCXB nature.

\subsection{SLX 1737-282}

SLX 1737-282 was first detected in 1985 with the Spacelab2 mission (Skinner et al. 1987) and was seen to radiate at the same flux level 5 times over the years (in 't Zand et al. 2002a). One burst was ever detected from this source despite large exposure times and the duration of the burst was extreme (the efolding decay time of $682 \mathrm{~s}$ is the longest ever measured apart from superbursts). The PCA bulge scan light curve is flat as well untill at least May 2005 after which it suffered source confusion from nearby transients. We performed an observation with the Swift X-Ray Telescope (XRT; Burrows et al. 2005) on Oct. 10, 2006, and find a $0.5-10 \mathrm{keV}$ unabsorbed flux of $(1.36 \pm 0.15) \times 10^{-10} \mathrm{erg} \mathrm{cm}^{-2} \mathrm{~s}^{-1}$, when fitting an absorbed power law in the $1-8 \mathrm{keV}$ band $\left(N_{\mathrm{H}}=(1.9 \pm 0.2) \times 10^{22} \mathrm{~cm}^{-2}\right.$, photon index $2.08 \pm 0.15$ and $\chi_{\text {red }}^{2}=0.6$ with 40 degrees of freedom), which is consistent with all previous measurements since discovery (cf., in 't Zand et al. 2002a).

No optical counterpart has been identified yet within the 8.' 3 (90\% confidence) ROSAT error circle radius (in 't Zand et al. 2003). The X-ray absorption column density of $N_{\mathrm{H}}=$ $1.9 \times 10^{22} \mathrm{~cm}^{-2}$ suggests a visual extinction of $A_{\mathrm{V}}=10.6$. Combined with the 5-8 kpc distance this brings the expected visual magnitude to $\approx 29$ for an ultracompact and $\approx 25$ for a nonultracompact binary. Confirmation of the UCXB nature through optical follow up may prove cumbersome.

\subsection{SLX 1744-299}

The nature of SLX 1744-299 (2:8 from another X-ray burster SLX 1744-300; Skinner et al. 1987, 1990) was established by Pavlinsky et al. (1994). They detected one long X-ray burst of several hundred seconds. Due to the small angular separation between both sources, all non-focusing X-ray telescopes are only able to measure the combined flux. A 2004 observation with XMM-Newton resolved both sources and found a 2.8/1.0 flux ratio in the 0.5-10 keV band between SLX 1744-299 and 


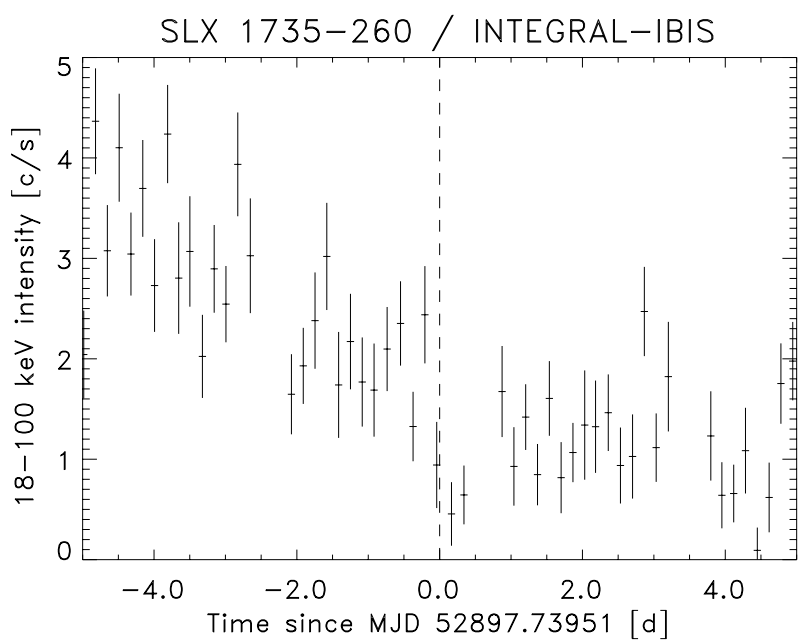

Fig. 9. The 18-100 keV light curve of SLX 1735-269 as measured around the occurrence of the very long X-ray burst on Sep. 15, 2003 (as indicated by the dashed line; Molkov et al. 2005) at a time resolution of 4 INTEGRAL science windows $(2.5 \mathrm{~h})$.

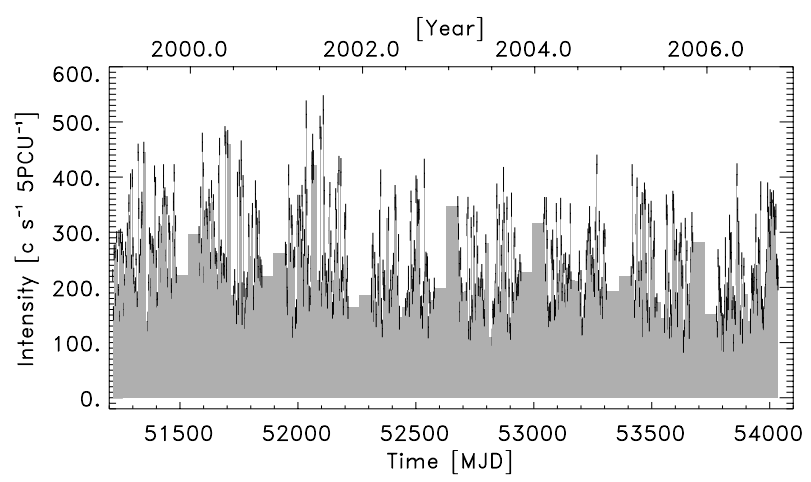

Fig. 10. The 2-12 keV light curve of SLX 1744-299 and 1744-300 combined as measured between February 1999 and November 2006 through the PCA bulge scan program.

SLX 1744-300 (Mori et al. 2005). This is the only such measurement. We applied this flux ratio to the ASM and PCA data in Table 2. The applicability of such a ratio is limited, since the PCA bulge scan light curve shows considerable variability by roughly a factor of two (Fig. 10).

BeppoSAX-WFC detected 48 bursts from both sources. Three of these are relatively long and can be identified with SLX 1744-299. The other 45 bursts are short and twice as faint. This is consistent with archival burst measurements which consistently reveal long and relatively bright bursts from SLX 1744299 and short and faint ones from SLX 1744-300. The longevity and slow recurrence of bursts from SLX 1744-299 are consistent with a UCXB nature.

\section{Confirmation of low $\dot{M}$ through a hard X-ray color}

Bird et al. (2006) present a catalog of 209 soft $\gamma$-ray sources detected with IBIS on INTEGRAL between February 2003 and June 2004. The catalog includes the average fluxes in 2 photon energy bands: $20-40$ and 40-100 keV. We made a selection of all 31 persistent $\mathrm{X}$-ray bursters in this list and ranked them according to their 40-100/20-40 keV hardness ratio, see Table 3. It includes 8 previously identified UCXBs and 3 new ones proposed here. The result is fascinating. Almost all of the UCXBs have
Table 3. Selection of all persistent X-ray bursters (ergo, LMXBs with a NS accretor) from the 2nd IBIS/ISGRI catalog (Bird et al. 2006), ranked according to increasing $40-100 / 20-40 \mathrm{keV}$ hardness ratio.

\begin{tabular}{|c|c|c|c|}
\hline Name & $\begin{array}{l}\text { Type } \\
\text { of src. }{ }^{1}\end{array}$ & $\begin{array}{r}40-100 \\
20-40 \\
\text { flux } \\
\text { ratio }\end{array}$ & UCXB? \\
\hline $4 \mathrm{U} 1735-444$ & $\overline{B, A}$ & $0.04 \pm 0.23$ & \\
\hline GX 17+2 & $\mathrm{B}, \mathrm{Z}$ & $0.05 \pm 0.09$ & \\
\hline GX 3+1 & $\mathrm{B}, \mathrm{A}$ & $0.06 \pm 0.25$ & \\
\hline 4U 1820-303 & $\mathrm{G}, \mathrm{B}, \mathrm{A}$ & $0.07 \pm 0.13$ & $\mathrm{y}$ \\
\hline Ser X-1 & B & $<0.09 \pm 0.02$ & \\
\hline Cyg X-2 & $\mathrm{B}, \mathrm{Z}$ & $<0.10 \pm 0.02$ & \\
\hline GX $13+1$ & $\mathrm{~B}, \mathrm{~A}$ & $0.29 \pm 0.06$ & \\
\hline GX 354-0 & $\mathrm{B}, \mathrm{A}$ & $0.38 \pm 0.01$ & \\
\hline 4U 1746-370 & $\mathrm{G}, \mathrm{B}, \mathrm{A}$ & $0.40 \pm 0.16$ & \\
\hline 4U 1915-05 & $\mathrm{B}, \mathrm{D}$ & $0.42 \pm 0.08$ & $\mathrm{y}$ \\
\hline 4U 1636-536 & $\mathrm{B}, \mathrm{A}$ & $0.55 \pm 0.03$ & \\
\hline 1A $1742-294$ & B & $0.56 \pm 0.03$ & \\
\hline 4U 1254-690 & $\mathrm{B}, \mathrm{D}$ & $<0.56 \pm 0.12$ & \\
\hline $4 \mathrm{U} 1705-440$ & $\mathrm{~B}, \mathrm{~A}$ & $0.60 \pm 0.02$ & \\
\hline 4U 1702-429 & $\mathrm{B}, \mathrm{A}$ & $0.62 \pm 0.03$ & \\
\hline 4U 1323-62 & $\mathrm{B}, \mathrm{D}$ & $0.63 \pm 0.17$ & \\
\hline SLX 1744-299 & B & $0.65 \pm 0.04$ & new \\
\hline 4U 1708-40 & B & $<0.67 \pm 0.17$ & \\
\hline 2S 0918-549 & B & $0.72 \pm 0.16$ & $\mathrm{y}$ \\
\hline GS 1826-24 & B & $0.80 \pm 0.01$ & \\
\hline SLX 1735-269 & B & $0.81 \pm 0.03$ & new \\
\hline 4U 1850-087 & $\mathrm{G}, \mathrm{B}$ & $0.83 \pm 0.09$ & y \\
\hline 4U 1722-30 & $\mathrm{G}, \mathrm{B}, \mathrm{A}$ & $0.88 \pm 0.02$ & new \\
\hline 4U 0614+091 & $\mathrm{B}, \mathrm{A}$ & $0.92 \pm 0.07$ & $\mathrm{y}$ \\
\hline XTE J1759-220 & $B, D$ & $0.93 \pm 0.05$ & \\
\hline 4U 1812-12 & B & $0.99 \pm 0.01$ & $\mathrm{y}$ \\
\hline 1A $1246-588$ & B & $1.00 \pm 0.25$ & $\mathrm{y}$ \\
\hline SLX 1737-282 & B & $1.03 \pm 0.07$ & new \\
\hline 4U 1705-32 & B & $1.03 \pm 0.12$ & $\mathrm{y}$ \\
\hline 1RXS J172525.2 & B & $1.11 \pm 0.15$ & new \\
\hline XTE J1710-281 & $\mathrm{B}, \mathrm{E}$ & $1.37 \pm 0.10$ & \\
\hline
\end{tabular}

${ }^{1} \mathrm{G}$ - in globular cluster; B - X-ray burster; A - Atoll source; Z - Z source; D - dipper; E - eclipser; ${ }^{2}$ full name 1RXS J172525.2-325717.

top rankings, constituting the hardest persistent X-ray bursters. There are 2 exceptions: 4U 1820-30 and 4U 1915-05.

The hard nature of the UCXBs is consistent with the low mass accretion rate inferred above (for a recent review of the spectral behavior versus mass accretion rate in NS LMXBs, see van der Klis 2006; see also Paizis et al. 2006). Therefore, this ranking is a confirmation of the low mass accretion rate. The two exceptions are also consistent with this. 4U 1820-30 has an accretion rate between 4 and $10 \times 10^{-9} M_{\odot} \mathrm{yr}^{-1}$ (Cumming 2003; note also that $P_{\text {orb }}$ (Stella et al. 1987) is the shortest of all). $4 \mathrm{U} 1915-05$ is a high-inclination system while none of the other UCXBs in Table 3 are and, therefore, probably a substantial multiplication factor is needed to correct for anisotropy of the persistent radiation and the system likely has a mass accretion rate of at least a few percent of Eddington. This may imply that it is similar to $4 \mathrm{U} 1820-303$ which may be supported by the fact that $4 \mathrm{U} 1915-05$ is the only UCXB to show a helium line in the optical spectrum (Nelemans et al. 2006b).

\section{Discussion}

In summary, we have ranked the estimated average mass accretion rate for all $40 \mathrm{X}$-ray bursters that have been active for at least 5 years and identified 16 cases with rates below $\approx 2 \%$ of the 
Eddington limit that do not have a high inclination angle. These include 9 UCXBs previously established on the basis of $P_{\text {orb }}$ measurements or low $L_{\mathrm{opt}} / L_{\mathrm{X}}$ values. We propose that the remaining eight cases are UCXBs as well, 2 of which have been proposed already in a preliminary study (in 't Zand et al. 2005a).

The correspondence between the low persistent flux and a low mass accretion rate is supported by 2 burst characteristics: 1 ) the recurrence times are relatively long: from 2.5 days (e.g., $4 \mathrm{U}$ 1722-30) up to at least 2 weeks (e.g., 1RXS J171824.2-402934, see in 't Zand et al. 2005a; see Table 2); 2) occasionally very long bursts are observed, falling just short of the superburst regime with e-folding decay times of up to $0.2 \mathrm{~h}$, which can be explained by a longer fuel accumulation time implied by the lower accretion rate and the cooler fuel temperature.

The long-term light curves of some newly identified UCXBs appear to exhibit a peculiar bimodal behavior with a slowly varying component (time scale hundreds of days) and a quickly varying component (time scale a few days). One newly identified UCXB, SLX 1735-269, shows an additional interesting feature in its light curve: an occasional complete drop of the $\mathrm{X}$-ray flux. Aside from these dips, variability of the same magnitude and time scales has been observed in (presumable) nonultracompact X-ray binaries as well, for instance in the bright sources GX 5-1, GX 9+1, GX 9+9, GX 340+0 and GX 349+2. The difference lies in the fact that the duty cycle of the fast component is much smaller in our UCXB candidates: the recurrence times of the flares in the fast component are of order tens of days in UCXB candidates while they are of order days in the bright non-ultracompact sources. It is tempting to suggest that this is due to a difference in mass ratio. For the UCXBs with pulsars, the mass ratio always is $q=M_{1} / M_{2}<0.1$ for probable inclination angles far from $0^{\circ}$ (see references in Table 1 ). It is well known that mass-transfering binaries with $q \lesssim 0.3$ are susceptible to tidal instabilities because the Kepler orbit around the accretor at which there is a 3:1 resonance between the Kepler frequency and binary orbit frequency then is inside the Roche lobe (Whitehurst 1988). This is thought to possibly result in an eccentric disk precessing with respect to the binary orbit which may modulate the accretion rate. In CVs this mechanism is thought to give rise to the so-called superoutbursts resulting from a combination of a thermal-viscous and a tidal instability in the disk. Perhaps in our systems only the tidal instability is active and the thermal-viscous instability is absent because the systems are persistent. The reason why SLX 1735-269 sometimes dies out completely is unclear. Obscuration by a warped disk appears an attractive explanation. We note that this behavior is not seen in all UCXBs. Possibly the effect is a sensitive function of $\dot{M}$, as suggested by the light curve of $4 \mathrm{U} 1722-30$ (Fig. 4). In conclusion, the peculiar light curve features point out possibly interesting implications for accretion disk theory.

A large, possibly dominant, fraction of LMXBs may be ultracompact. If the 6 new UCXBs are valid, 18 out of the 40 persistent bursters are ultracompact. Possibly several more are ultracompact, because not all remaining systems yet have optical counterparts through which an ultracompact nature may be indicated; some could be similar to $4 \mathrm{U} 1820-303$. The fraction of ultracompact cases is $7 / 8$ in globular clusters (4U 1746-37 being the sole exception) and between 11/32 and 21/32 in the Galactic field (the upper limit being defined by the 11 LMXBs with measured non-ultracompact periods). The numbers are too small to analyze differences between the globular cluster and Galactic field populations in a meaningful way.

Ultimate verification of an ultracompact nature can only be done through measurement of $P_{\text {orb}}$. The most succesful (tentative) measurements were done through optical photometry. Many of the candidate UCXBs are located near the Galactic center, implying large extinctions of at least $5 \mathrm{mag}$ in $V$. Combined with a distance modulus of 14.5 (for a canonical $8 \mathrm{kpc}$ distance) and an expected $M_{V}$ of about 4 (van Paradijs \& McClintock 1994), renders photometry towards the infrared with $6 \mathrm{~m}$ class telescopes as the only possible route.

Acknowledgements. We are grateful to Andrew Cumming, Gijs Nelemans, Duncan Galloway, Michael Truss, Jean-Pierre Lasota and Laurens Keek for very useful discussions, Lucien Kuiper and Peter den Hartog for help with understanding INTEGRAL data, Sergey Molkov for providing the processed INTEGRAL data on the burst from SLX 1735-269 and Frank Verbunt for providing useful comments on an earlier version of the manuscript. We thank the Swift team for granting and preparing a target of opportunity observation of SLX 1737-282 to clarify our flux measurements with the PCA bulge program. This research has made use of the RXTE/ASM archive provided by ASM teams at MIT and at the RXTE SOF and GOF at NASA/GSFC, of the INTEGRAL data archive provided by the INTEGRAL Science Data Center at the Observatoire de Genève, and of data from the Italian-Dutch BeppoSAX mission provided by the ASI Science Data Center in Rome. J.Z. acknowledges support from The Netherlands Organization for Scientific Research (NWO).

\section{References}

Bassa, C. G., Jonker, P. G., in 't Zand, J. J. M., \& Verbunt, F. 2006, A\&A, 446, L17

Barret, D., Olive, J. F., \& Oosterbroek, T. 2003, A\&A, 400, 643

Bazzano, A., Cocchi, M., Ubertini, P., et al. 1997, IAUC, 6668

Bildsten, L. 1998, in The Many Faces of Neutron Stars., ed. R. Buccheri, J. van Paradijs, \& A. Alpar, NATO ASIC Proc., 515, 419

Bird, A. J., Barlow, E. J., Bassani, L., et al. 2006, ApJ, 636, 765

Brandt, S., Budtz-Jørgensen, \& Chenevez, J. 2006, ATel, 778

Burrows, D. N., Hill, J. E., Nousek, J. A., et al. 2005, SSRv, 120, 165

Chelovekov, I. V., Grebenev, S. A., \& Sunyaev, R. A., 2006, AstL, 32, 456 [arXiv: astro-ph/0605638]

Chevalier, C., \& Ilovaisky, S. A. 1990, A\&A, 228, 115

Cocchi, M., Natalucci, L., in 't Zand, J. J. M., et al. 1999, IAUC, 7247

Cocchi, M., Bazzano, A., Natalucci, L., et al. 2000a, A\&A, 357, 527

Cocchi, M., Bazzano, A., Natalucci, L., et al. 2000b, Proc. 5th Compton Symp., AIP, 510, 217

Cocchi, M., Bazzano, A., Natalucci, L., Heise, J., \& in 't Zand, J. J. M. 2001, Mem. S.A.It., 72, 757

Cornelisse, R., Verbunt, F., In 't Zand, J. J. M., et al. 2002a, A\&A, 392, 885

Cornelisse, R., Verbunt, F., In 't Zand, J. J. M., Kuulkers, E., \& Heise, J. 2002b, A\&A, 392, 931

Cornelisse, R., In 't Zand, J. J. M., Verbunt, F., et al. 2003, A\&A, 405, 1033 Cumming, A. 2003, ApJ, 595, 1077

Cumming, A., Macbeth, J., in 't Zand, J. J. M., \& Page, D. 2006, ApJ, 646, 429 Deloye, C. J., \& Bildsten, L. 2003, ApJ, 598, 1217

Deutsch, E. W., Margon, B., \& Anderson, S. F. 2000, ApJ, 530, L21

Dickey, J. M., \& Lockman, F. J. 1990, ARA, 28, 215

Dieball, A., Knigge, C., Zurek, D. R., et al. 2005, ApJ, 634, L105

Dubus, G., Lasota, J.-P., Hameury, J.-M., \& Charles, P. 1999, MNRAS, 303, 139

Elvis, M., Plummer, D., McDowell, J., \& Bechtold, J. 1992, ApJS, 80, 257

Fabian, A. C., Pringle, J. E., \& Rees, M. J. 1975, Nature, 255, 208

Fujimoto, M. Y. 1988, ApJ, 324, 995

Fujimoto, M. Y., Hanawa, T., \& Miyaji, S. 1981, ApJ, 247, 267

Galloway, D., Chakrabarty, D., Morgen, E. H., \& Remillard, R. A. 2002, 576, L137

Galloway, D. K., Psaltis, D., Chakrabarty, D., \& Muno, M. P. 2003, ApJ, 590, 999

Galloway, D. K., Cumming, A., Kuulkers, E., et al. 2004, ApJ, 601, 466

Galloway, D., Muno, M. P., Chakrabarty, D., et al. 2006, ApJS, submitted [arXiv: astro-ph/0608259]

Haggard, D., Cool, A. M., Anderson, J., et al. 2004, ApJ, 613, 512

Hakala, P., Ramsay, G., Muhli, P., et al. 2005, MNRAS, 356, 1133

Heinke, C. O., Edmonds, P. D., \& Grindlay, J. E. 2001, ApJ, 562, 363

Heinke, C. O., Edmonds, P. D., Grindlay, J. E., et al. 2003, ApJ, 590, 809

Hoffman, J. A., Cominsky, L., \& Lewin, W. H. G. 1980, ApJ, 240, L27

Homer, L., Charles, P. A., Naylor, T., et al. 1996, MNRAS, 282, L37

Hoshi, R. 1979, Progress Th. Phys., 61, 1307

Hynes, R. I., Robinson, E. L., \& Jeffery, E. 2004, ApJ, 608, L101

Iaria, R., Di Salvo, T., Burderi, L., \& Robba, N. R. 2001, ApJ, 548, 883 
in 't Zand, J. J. M., Heise, J., Bazzano, A., Cocchi, M., \& Smith, M. J. S. 1999, IAUC, 7243

in 't Zand, J. J. M., Verbunt, F., Kuulkers, E., et al. 2002a, A\&A, 389, L43

in 't Zand, J. J. M., Markwardt, C. B., Bazzano, A., et al. 2002b, A\&A, 390, 597

in 't Zand, J. J. M., Kuulkers, E., Verbunt, F., \& Cornelisse, R. 2003, A\&A, 411, $\mathrm{L} 487$

in 't Zand, J. J. M., Verbunt, F., Heise, J., et al. 2004, Nucl. Phys. B. Proc. Ser., 132,486

in 't Zand, J. J. M., Cornelisse, R., \& Méndez, M. 2005a, A\&A, 440, 287

in 't Zand, J. J. M., Cumming, A., van der Sluys, M. V., Verbunt, F., \& Pols, O. R. 2005b, A\&A, 441, 675

Jonker, P. G., Bassa, C. G., Nelemans, G., et al. 2006, MNRAS, 368, 1803

Juett, A. M., \& Chakrabarty, D. 2003, ApJ, 599, 498

Juett, A. M., \& Chakrabarty, D. 2005, ApJ, 627, 926

Juett, A. M., Psaltis, D., \& Chakrabarty, D. 2001, ApJ, 560, L59

Kaptein, R. G., in 't Zand, J. J. M., Kuulkers, E., et al. 2000, A\&A, 358, L71

Kirsch, M. G., Briel, U. G., Burrows, D., et al. 2005, in Proc. UV, X-Ray, and Gamma-Ray Space Instrumentation for Astronomy XIV, ed. O. H. W. Siegmund, Proc. SPIE, 5898, 22

Kuulkers, E., \& van der Klis, M. 2000, A\&A, 356, L45

Kuulkers, E., Homan, J., van der Klis, M., Lewin, W. H. G., \& Méndez, M. 2002, A\&A, 382, 503

Kuulkers, E., den Hartog, P. R., in 't Zand, J. J. M., et al. 2003, A\&A, 399, 663

Lapidus, I. I., \& Sunyaev, R. A. 1985, MNRAS, 217, 291

Levine, A., Bradt, H., Cui, W., et al. 1996, ApJ, 469, L33

Lewin, W. H. G., van Paradijs, J., \& Taam, R. E. 1993, SSRv, 62, 223

Markwardt, C. B. 2006, in Proc. The transient Milky Way: a perspective for MIRAX, ed. F. D’Amico, J. Braga, \& R. E. Rothschild, AIP Conf. Proc., 840,45

Markwardt, C. B., Swank, J. H., Strohmayer, T. E., in 't Zand, J. J. M., \& Marshall, F. E. 2002, ApJ, 575, L21

Markwardt, C. B., Juda, M., \& Swank, J. H. 2003, ATel, 127

Matsuba, E., Dotani, T., Mitsuda, K., et al. 1995, PASJ, 47, 575

Menou, K., Perna, R., \& Hernquist, L. 2002, ApJ, 564, L81

Middleditch, J., Mason, K. O., Nelson, J. E., \& White, N. E. 1981, ApJ, 244, 1001

Migliari, S., Di Salvo, T., Belloni, T., et al. 2003, MNRAS, 342, 909

Molkov, S., Grebenev, S. A., \& Lutovinov, A. A. 2000, A\&A, 357, L41

Molkov, S., Revnivtsev, M., Lutovinov, A., \& Sunyaev, R. A. 2005, A\&A, 434, 1069

Mori, H., Maeda, Y., Pavlov, G., Sakano, M., \& Tsuboi, Y. 2005, AdSpR, 35, 1137

Nelemans, G., Jonker, P. G., Marsh, T. R., van der Klis, M. 2004, MNRAS, 348, L7

Nelemans, G., \& Jonker, P. 2006a, in A Life with Stars, Symposium, Amsterdam, August 2005, ed. L. Kaper, M. van der Klis, \& R. A. M. J. Wijers, in press [arXiv: astro-ph/0605722]

Nelemans, G., Jonker, P., \& Steeghs, D. 2006b, MNRAS, 370, 255

Nelson, L. A., Rappaport, S. A., \& Joss, P. C. 1986, ApJ, 304, 231

O'Brien, K. 2005, presentation at symposium, A Life With Stars, Amsterdam

Ortolani, S., Bica, E., \& Barbuy, B. 1997, A\&A, 326, 614
Osaki, Y. 1974, PASJ, 26, 429

Osaki, Y. 1996, PASP, 108, 39

Paizis, A., Farinelli, R., Ittarchuk, L., et al. 2006, A\&A, in press [arXiv: astro-ph/0607592]

Parmar, A. N., Oosterbroek, T., Sidoli, L., Stella, L., \& Frontera, F. 2001, A\&A, 380,490

Pavlinsky, M. N., Grebenev, S. A., \& Sunyaev, R. A. 1994, ApJ, 425, 110

Peng, F., Brown, E., \& Truran, J. 2006, ApJ, submitted

Savonije, G. J., de Kool, M., \& van den Heuvel, E. P. J. 1986, A\&A, 155, 51

Schatz, H., Aprahamian, A., Barnard, V., et al. 2001, Phys. Rev. Lett., 86, 3471

Sidoli, L., Parmar, A. N., Oosterbroek, T., et al. 2001, A\&A, 368, 451

Skinner, G. K., Willmore, A. P., Eyles, C. J., Bertram, D., \& Church, M. J. 1987, Nature, 330, 544

Skinner, G. K., Foster, A. J., Willmore, A. P., \& Eyles, C. J. 1990, MNRAS, 243 , 72

Smak, J. 1983, ApJ, 272, 234

Smale, A.P., Corbet, R. H. D., Charles, P. A., et al. 1988, MNRAS, 233, 51

Stella, L., Priedhorsky, W., \& White, N. E. 1987, ApJ, 312, L17

Stephen, J. B., Bassani, L., Molina, M., et al. 2005, A\&A, 432, L49

Strohmayer, T. E., \& Bildsten, L. 2006, in Compact Stellar X-ray Sources, ed. W. H. G. Lewin, \& M. van der Klis, CUP (Cambridge, UK), 113

Suzuki, M., \& Kawai, N. 2005, ATel, 539

Swank, J. H., \& Markwardt, C. B. 2001, in Populations of Transient Galactic Bulge X-ray Sources, New Century of X-ray Astronomy, ed. H. Inoue, \& H. Kunieda, ASP Conf. Ser., 251, 94

Swank, J. H., Becker, R. H., Pravdo, S. H., Saba, J. R., \& Serlemitsos, P. J. 1976, IAUC, 3010

Swank, J., Becker, R. H., Boldt, E. A., Holt, S. S., \& Serlemitsis, P. J. 1978, MNRAS, 182, 349

Tennant, A., Fabian, A. C., \& Shafer, R. A. 1986, MNRAS, 221, 27

Van der Klis, M. 2006, in Compact Stellar X-ray Sources, ed. W. H. G. Lewin, \& M. van der Klis, CUP (Cambridge, UK), 39

van Paradijs, J. 1996, A\&A, 464, L139

Van Paradijs, J., \& McClintock, J. E. 1994, A\&A, 290, 133

van Paradijs, J., Dotani, T., Tanaka, Y., \& Tsuru, T. 1990, PASJ, 42, 633

Verbunt, F., \& Lewin, W. H. G. 2006, in Compact Stellar X-ray Sources, ed. W. H. G. Lewin, \& M. van der Klis, CUP (Cambridge, UK), 341

Walter, R., Bodaghee, A., BArlow, E. J., et al. 2004, ATel, 229

Wang, Z., \& Chakrabarty, D. 2004, ApJ, 616, L139

Wen, L., Levine, A., Corbet, R. H. D., \& Bradt, H. V. 2006, ApJS, 163, 372

Werner, K., Nagel, T., Rauch, T., Hammer, N. H., \& Dreizler, S. 2006, A\&A, 450,725

White, N. E., \& Angelini, L. A. 2001, ApJ, 561, L101

White, N. E., \& Swank, J. H. 1982, ApJ, 253, L61

White, N. E., Kaluzienski, J. L., \& Swank, J. H. 1984, in High Energy Transients in Astrophysics, ed. S. E. Woosley, AIP Conf. Proc. 115, 31

Whitehurst, R. 1988, MNRAS, 232, 35

Wilson, C. A., Patel, S. K., Kouvelioutou, C., et al. 2003, ApJ, 596, 1220

Wolff, M. T., Becker, P. A., Ray, P. S., \& Wood, K. S. 2005, ApJ, 632, 1099

Woosley, S., Heger, A., Cumming, A., et al. 2004, ApJS, 151, 75 